\title{
A generative flow-based model for volumetric data augmentation in 3D deep learning for computed tomographic colonography
}

\author{
Tomoki Uemura ${ }^{1,2}$ - Janne J. Näppi ${ }^{1} \cdot$ Yasuji Ryu $^{3} \cdot$ Chinatsu Watari ${ }^{1} \cdot$ Tohru Kamiya $^{2} \cdot$ Hiroyuki Yoshida $^{1}$
}

Received: 30 April 2020 / Accepted: 30 September 2020 / Published online: 5 November 2020

(c) The Author(s) 2020

\begin{abstract}
Purpose Deep learning can be used for improving the performance of computer-aided detection (CADe) in various medical imaging tasks. However, in computed tomographic (CT) colonography, the performance is limited by the relatively small size and the variety of the available training datasets. Our purpose in this study was to develop and evaluate a flow-based generative model for performing 3D data augmentation of colorectal polyps for effective training of deep learning in CADe for CT colonography.

Methods We developed a 3D-convolutional neural network (3D CNN) based on a flow-based generative model (3D Glow) for generating synthetic volumes of interest (VOIs) that has characteristics similar to those of the VOIs of its training dataset. The 3D Glow was trained to generate synthetic VOIs of polyps by use of our clinical CT colonography case collection. The evaluation was performed by use of a human observer study with three observers and by use of a CADe-based polyp classification study with a 3D DenseNet.

Results The area-under-the-curve values of the receiver operating characteristic analysis of the three observers were not statistically significantly different in distinguishing between real polyps and synthetic polyps. When trained with data augmentation by 3D Glow, the 3D DenseNet yielded a statistically significantly higher polyp classification performance than when it was trained with alternative augmentation methods.

Conclusion The 3D Glow-generated synthetic polyps are visually indistinguishable from real colorectal polyps. Their application to data augmentation can substantially improve the performance of 3D CNNs in CADe for CT colonography. Thus, 3D Glow is a promising method for improving the performance of deep learning in CADe for CT colonography.
\end{abstract}

Keywords Generative models · Data augmentation - Deep learning · Computer-aided detection · Virtual colonoscopy · Artificial intelligence

\section{Introduction}

Colorectal cancer (CRC) is the third most common cancer in terms of incidence and the second most common cancer

Tomoki Uemura and Janne J. Näppi contributed equally to this work.

Hiroyuki Yoshida

yoshida.hiro@mgh.harvard.edu

1 3D Imaging Research, Department of Radiology, Massachusetts General Hospital and Harvard Medical School, 25 New Chardon Street, Suite 400C, Boston, MA 02114, USA

2 Department of Mechanical and Control Engineering, Kyushu Institute of Technology, 1-1 Sensui-cho, Kitakyushu 804-8550, Japan

3 Department of Radiology, Tonami General Hospital, 1-61 Shintomi-cho, Tonami, Toyama 939-1395, Japan in terms of mortality worldwide [1]. However, CRC can be prevented considerably by early detection and removal of its precursors, colorectal polyps [2]. Computed tomographic colonography (CTC) can be used both for detecting CRCs and for preventing CRCs by early detection of clinically significant polyps that could develop into cancers [3].

Deep learning based on convolutional neural networks (CNNs) has made it easy to obtain state-of-the-art results in various medical imaging tasks [4]. However, one of the limitations of deep learning is that the development of generalizable CNNs requires a large amount and great variety of training data [5]. In CTC, the available training datasets are relatively small and limited in numbers and variations.

The two principal approaches for addressing the issue of small training datasets in deep learning have included (1) transfer learning of CNNs and (2) data augmentation 
of training datasets. With the first approach, transfer learning, one starts with a CNN that has been pre-trained with a large number of images. The $\mathrm{CNN}$ is then adapted to the desired application by continuation of the training with the available domain-specific data. In CTC, transfer learning has been used successfully in training of CNNs for interpreting virtual endoluminal views [6], for detecting contrast-coated serrated polyps [7] and for performing electronic cleansing [8]. However, transfer learning has the limitations that most of the publicly available pre-trained CNNs have not been pretrained with medical images and that most of these CNNs are 2D CNNs.

With the second approach, data augmentation, the training dataset is enhanced artificially so that the number and variety of training samples are increased. The most common method for implementing data augmentation has been the manipulation of the training samples by basic image processing operations [5]. In CTC, this approach has been used successfully for training of CNNs for detection of polyps [9] and masses [10], and for reduction in the number of falsepositive (FP) detections in computer-aided detection (CADe) $[11,12]$. However, the approach has the limitation that some of the image transforms that are commonly used with natural images are not appropriate for use with medical images. This can limit the number and variety of the obtainable training samples.

Recently, several studies have explored the possibility of performing data augmentation by use of generative adversarial networks (GANs) [13,14]. In CTC, 3D GANs have been used for generation of synthetic polyps to improve the training of 3D CNNs in CADe [15]. However, the development of GANs that can generate realistic synthetic images at a high image resolution is known to suffer from various problems, such as non-convergence of the model parameters, mode collapse or training imbalance between the generator and the discriminator [5].

Flow-based generative models have several advantages over GANs [16]. Unlike GANs, they are designed to learn the distribution of the input data explicitly, thereby providing an exact latent-variable inference, log-likelihood evaluation and a meaningful latent space for performance of valid manipulations of the data. They are also highly parallelizable and can be implemented with a small memory footprint [16].

In this study, we investigated the feasibility of applying a flow-based generative model to 3D data augmentation of colorectal polyps in CTC. We hypothesized that (1) a 3D CNN that implements a state-of-the-art flow-based generative model known as Glow [16] (hereafter referred to as 3D Glow) can be trained to generate synthetic polyps that are visually indistinguishable from real colorectal polyps in CTC, and that (2) data augmentation by use of 3D Glowgenerated synthetic polyps can be used for improving the performance of deep learning in differentiating between polyps and non-polyps in CADe for CTC. One could use successful development of 3D Glow to overcome the limitations of currently available CTC datasets, thereby enabling the training of generalizable high-performing 3D CNNs for CADe in CTC.

\section{Flow-based generative model}

Given an observed (complicated) probability distribution, a flow-based generative model provides a bijective mapping $f$ between the observed distribution and a simple, wellunderstood target probability distribution, such as a standard Gaussian distribution. The desired computations can then be performed on the simple target distribution rather than on the observed distribution. Such a mapping that is constructed as a sequence of invertible bijective transforms is called the normalizing flow. Formally, let $x$ denote an observation sampled from a training dataset $D$ that has an unknown probability distribution $p(x)$. The normalizing flow can be defined as

$z=f(x)=f_{L} \circ f_{L-1} \circ \cdots \circ f_{1}(x)$,

where $z$ is a latent random variable of the desired target distribution. The corresponding flow-based generative model is constructed by optimizing the parameters of the component functions of Eq. (1), $f_{i}$, by use of the maximum-likelihood method. The training loss is the negative log-likelihood over $D$, or

$L(D)=-\frac{1}{|D|} \sum_{x \in D} \log p(x)$.

Figure 1 illustrates the construction of a normalizing flow. The path traversed by the random variables, $z_{i}$, is a flow, and the full chain that is formed by the corresponding probability distributions is the normalizing flow.

\section{D Glow}

Figure 2a illustrates the overall architecture of our 3D Glow model. The model extends the originally proposed 2D Glow framework [16] into a 3D CNN for processing of volumetric CTC images.

The model has three types of layer blocks. Squeeze transforms an input $w \times h \times d \times c$ tensor, where $w \times h \times d$ is the input image size and $c$ is the number of channels, into a $\frac{w}{2} \times \frac{h}{2} \times \frac{d}{2} \times 8 c$ tensor; thus, trading spatial size for number of channels [17]. Flow implements one step of the flow. Split splits the input tensor along the channel dimensions [16].

Figure $2 \mathrm{~b}$ illustrates the design of a Flow block. There are three layers: an activation-normalization (actnorm) layer 
Fig. 1 Diagram of a normalizing flow between a simple Gaussian distribution $z_{0} \sim p_{0}\left(z_{0}\right)$ and an observed distribution $x=z_{L} \sim p_{L}\left(z_{L}\right)$
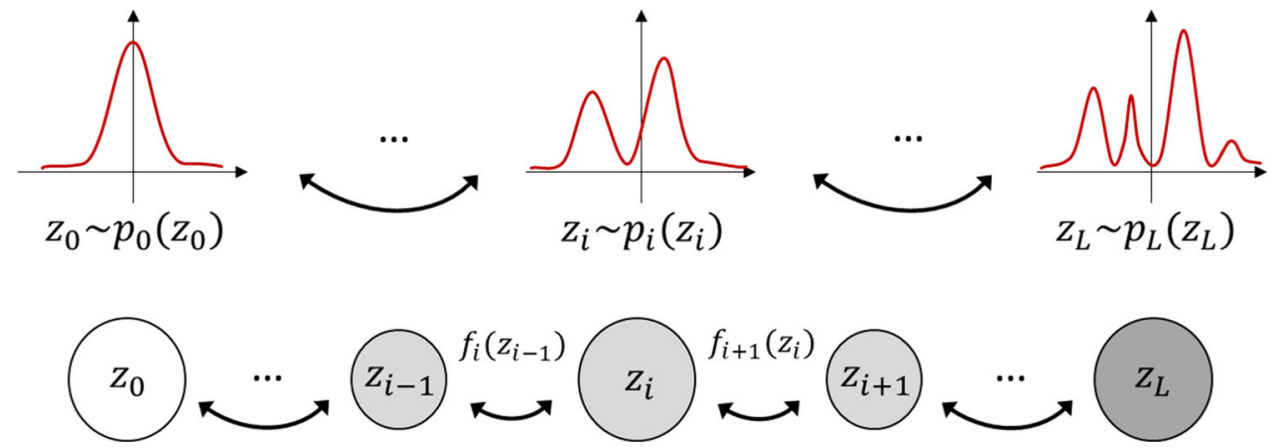

3D Glow

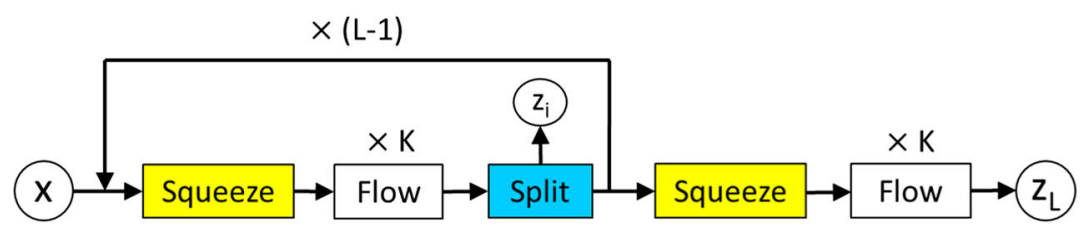

(a)

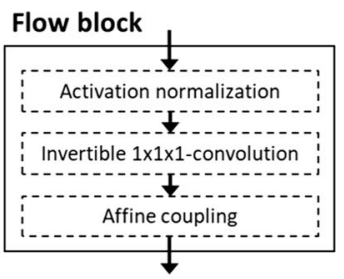

(b)

Fig. 2 a Illustration of the architecture of 3D Glow. The model implements $K$ levels and $L$ steps of flow [16]. b Design of a flow block

[16], an invertible $1 \times 1 \times 1$-convolutional layer [16] and an affine-coupling layer [18]. The actnorm layer performs an affine transformation of the input tensor, where the trainable per-channel scale and bias parameters are initialized to yield a mean of zero and a standard deviation of one based on the first mini-batch [16]. The invertible $1 \times 1 \times 1$-convolutional layer implements a trainable permutation that reverses the order of the input channels, where the weight matrix of the transform is initialized as a random rotation matrix [16]. The affine-coupling layer splits the input into two parts: the first $d$ dimensions remain the same, whereas the latter dimensions, $d+1$ to $D$, undergo an affine transformation based on the scale and shift parameters as

$$
\begin{aligned}
y_{1: d} & =x_{1: d}, \\
y_{d+1: D} & =x_{d+1: D} \odot \exp \left(s\left(x_{1: d}\right)\right)+t\left(x_{1: d}\right),
\end{aligned}
$$

where $\odot$ denotes an element-wise product and $s($.$) and t($.) are scale and translation functions that map $\mathbb{R}^{d} \rightarrow \mathbb{R}^{D-d}$.

It can be shown that the transformation above satisfies the basic properties required by efficient computation of the flow transformation [16]. The model is trained with the loss function of Eq. (2).

\section{Construction of synthetic polyps}

Figure 3 illustrates the principle of the 3D Glow-based data augmentation. The training of the model involves the learning of a bijective mapping from input polyp VOIs (volumes of interest, $x_{a}$ and $\left.x_{b}\right)$ to vectors of the latent space $\left(z_{a}\right.$ and $\left.z_{b}\right)$ by use of the latent variable $z$ and the normalizing flow of Eq. (1).

We interpolate the new samples in the latent space by use of linear interpolation between any two training samples. Given two vectors of the latent space, such as $z_{a}$ and $z_{b}$, we calculate a new sample as $\tilde{z} \sim z_{a}+\alpha\left(z_{b}-z_{a}\right)$, where $\alpha$ is sampled randomly from the uniform distribution and scaled linearly to $\alpha \in[0.4,0.6]$ to avoid generating VOIs that would be nearly identical to the VOI of real polyps in the training dataset.

\section{Materials}

\section{CTC cases}

We constructed two independent CTC datasets for the experiments: a development dataset and a test dataset. The development dataset was constructed for providing samples of polyps for the training of 3D Glow for generating synthetic polyps and for providing samples of real polyps and normal anatomy for training of a 3D CNN for polyp detection. The independent test dataset was constructed for testing of the 3D CNN performance. The details of the training and testing of the polyp detection are explained in the section of "Polyp classification study" in "Evaluation methods" section.

We constructed a retrospective development dataset of 203 patients with 269 colonoscopy-confirmed polyps $\geq 6 \mathrm{~mm}$ in size from cases of various clinical CTC screening trials [1922]. Most of the patients had been prepared with a cathartic bowel preparation and oral contrast (fecal tagging) by iodine 


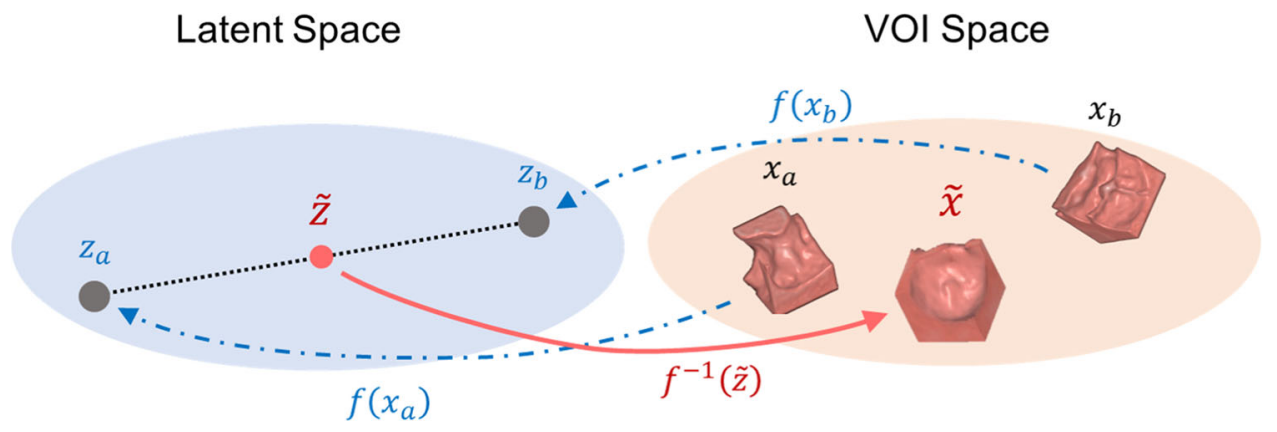

Fig. 3 Illustration of 3D Glow-based data augmentation. Two training VOIs, $x_{a}$ and $x_{b}$, are mapped to points $z_{a}$ and $z_{b}$ of the latent space, which are used for interpolating a new point $\tilde{z}$. By mapping $\tilde{z}$ back to the VOI space, we generate a new synthetic VOI, $\tilde{x}$, which is different from, yet similar to $x_{a}$ and $x_{b}$

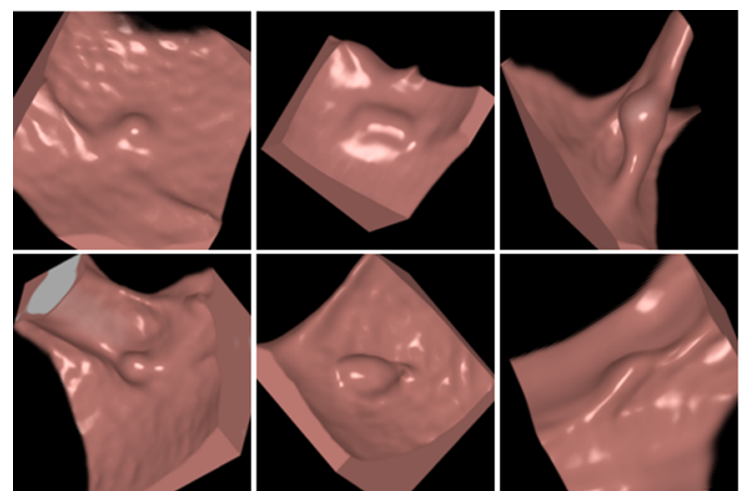

(a)

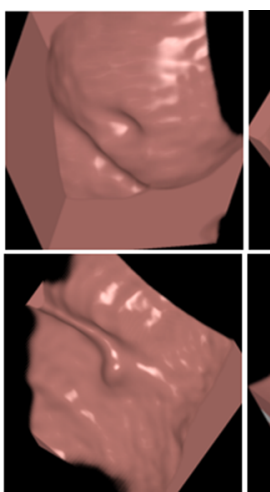

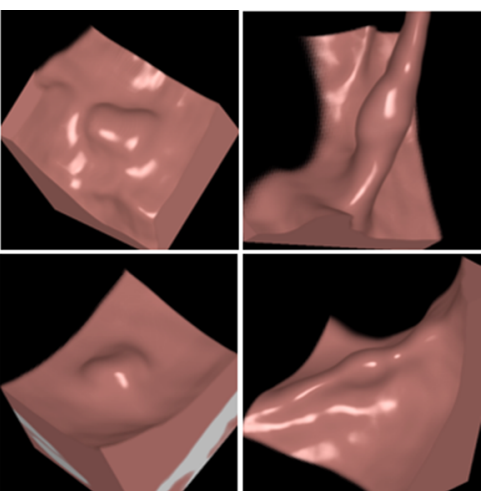

(b)

Fig. 4 Virtual endoscopic views of the VOIs of a six real polyps and $\mathbf{b}$ six 3 D Glow-generated synthetic polyps. Each VOI was $32 \times 32 \times 32$ voxels in size

and/or barium. The patients had been scanned in two (supine, prone and/or decubitus) positions by use of a total of $11 \mathrm{CT}$ scanner models (Siemens, Philips, Toshiba, and GE Medical Systems) at 120 or $140 \mathrm{kVp}, 0.54-0.97 \mathrm{~mm}$ pixel spacing, $0.75-5.00 \mathrm{~mm}$ slice thickness and $0.7-2.5 \mathrm{~mm}$ reconstruction interval. The spatial locations of the polyps on the CTC images were established by experienced radiologists.

We constructed an independent test dataset of 36 patients with 64 colonoscopy-confirmed polyps from two distinct subgroups of patients. The first subgroup of these patients (20 patients with 45 polyps) was prepared by use of a reduced cathartic bowel preparation with $18 \mathrm{~g}$ of magnesium citrate and fecal tagging by $50 \mathrm{ml}$ of non-ionic iodine. The CTC acquisitions (SOMATOM Definition, Siemens Healthcare, Erlangen, Germany) were performed at $140 \mathrm{kVp}$ with $64 \times 0.6 \mathrm{~mm}$ collimation, pitch of 0.85 , rotation time of $0.5 \mathrm{~s}$, $1.0 \mathrm{~mm}$ slice thickness and a $0.6-0.7 \mathrm{~mm}$ reconstruction interval. The second subgroup (16 patients with 19 polyps) was prepared by use of the previously established protocol of polyethylene glycol solution plus contrast medium [23]. The CTC acquisitions (Acquilion, Canon Medical Systems, Tochigi, Japan) were performed at $120 \mathrm{kVp}$ with 16 or $64 \times 0.6 \mathrm{~mm}$ collimation, pitch of $0.81-0.94$, rotation time of $0.5 \mathrm{~s}, 0.5$ or $1.0 \mathrm{~mm}$ slice thickness and 0.8 or $1.0 \mathrm{~mm}$ reconstruction intervals.

\section{Extraction of VOls}

For the experiments, we extracted three types of VOIs by use of the development dataset: 100 VOIs of real polyps, 100 VOIs of synthetic polyps and 100 VOIs of normal anatomy. The $100 \mathrm{VOIs}$ of real polyps were those of randomly sampled real polyps. The VOIs of synthetic polyps were generated by 3D Glow after training with the VOIs of the 269 real polyps from the development dataset. The VOIs of normal anatomy were the VOIs of randomly sampled FP detections obtained at $100 \%$ polyp detection sensitivity by the $3 \mathrm{D}$ detection module of our previously developed CADe system [24-26].

Figure 4 shows examples of the VOIs of the real polyps and 3D Glow-generated synthetic polyps that were included in our experiments. The synthetic polyps look visually indistinguishable from real polyps. Figure 5 shows a visual comparison of the radiomic values of the polyp regions of the real and synthetic polyps in terms of two radiomic features that we had previously identified as being most effective in 


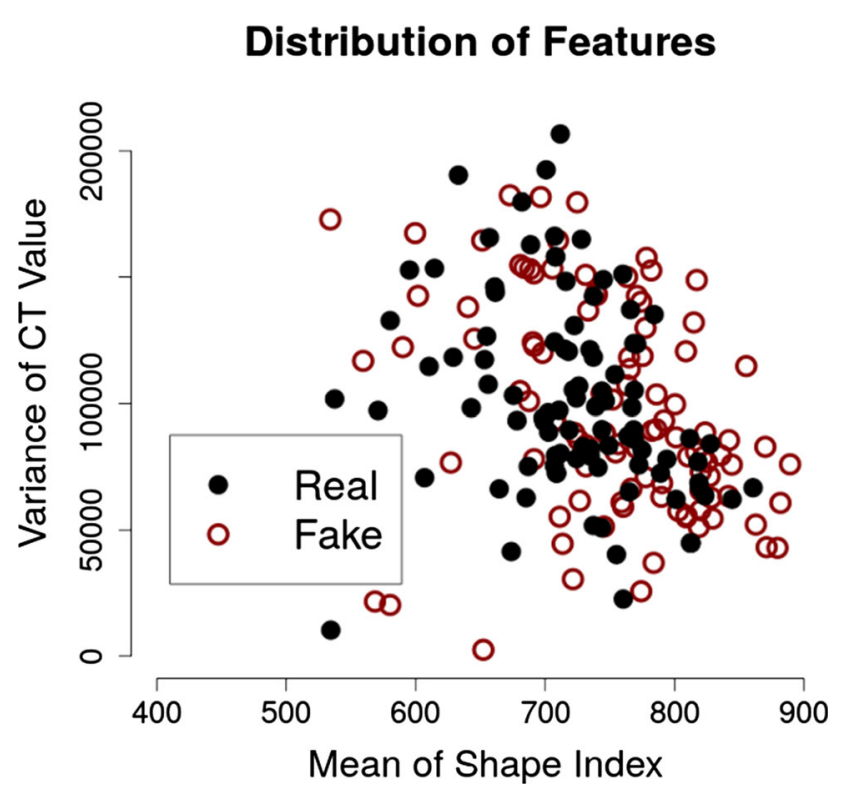

Fig. 5 Visual comparison of the volumetric shape and texture features of the 100 real polyps ("Real") and 100 3D Glow-generated synthetic polyps ("Fake")

the differentiation of true polyps from non-polyps in CADe [27]. The comparison indicates that the synthetic and real polyps are radiomically indistinguishable.

For independent testing, we acquired all of the VOIs of the polyp candidates that were detected from the test dataset (see the subsection of "CTC cases") by the detection module of our previously developed CADe system. There were 92 true-positive (TP) detections $\geq 6 \mathrm{~mm}$ in their largest diameter (6-9 mm: 61 TPs) and 6519 FP detections. To calculate meaningful area-under-the-curve (AUC) values for receiver operating characteristic (ROC) analysis, we balanced the number of TP and FP detections in the test dataset by data augmentation of the TP VOIs with 3D rotations. This yielded a total of 12,407 VOIs for independent testing.

\section{Evaluation methods}

\section{Observer study}

Three experienced observers (two physicians and one physicist, each with experience of reading $>500$ CTC studies) attempted to differentiate the VOIs of the 100 real polyps from those of the 100 3D Glow-generated synthetic polyps. The VOIs were loaded to a commercial CTC reading workstation (AZE Virtual Place Raijin, Canon Inc., Tokyo, Japan), where they were presented to the readers in random order. The readers were instructed to evaluate the VOIs interactively by use of the standard 2D and 3D CTC reading modes. For each VOI, the reader recorded his/her confidence that the VOI contained a real polyp, on a scale from 0 (synthetic polyp) to 10 (real polyp). The reading time was unlimited.

The discrimination performance was evaluated by use of ROC analysis, where the confidence levels recorded by each reader were analyzed by use of the pROC package (version 1.16.2) [28] in R (version 3.6.3) [29]. The ROC curves were generated by use of binomial fitting. The $95 \%$ confidence intervals were computed by use of bootstrapping with 1000 replicates. The difference of the AUC value from $50 \%$ (the level of not being able to tell the difference between a real polyp and a synthetic polyp) was tested by use of a bootstrap test with 1000 replicates, where $p<0.05$ indicated a statistically significant difference.

\section{Polyp classification study}

We trained our previously developed 3D DenseNet CNN [30] to detect polyps with five data augmentation approaches: baseline augmentation, nonlinear augmentation, 3D GAN with baseline augmentation, 3D Glow with baseline augmentation and 3D Glow with nonlinear augmentation. The baseline augmentation included random flipping and/or 13-times zooming of the VOIs. The nonlinear augmentation included the baseline augmentation plus 3D shifting, 3D rotation and/or application of Gaussian noise to the CT values of the VOIs. The GAN-based augmentation was based on our previously developed 3D self-attention GAN method for generating synthetic 3D polyp VOIs [15].

For the training of the 3D DenseNet, 200 VOIs of real polyps and normal anatomy were extracted from the development dataset as described in section "Extraction of VOIs". In the baseline and nonlinear augmentation approaches, the 3D DenseNet was trained with the 200 VOIs augmented by each approach. In the Glow- and GAN-based augmentations, the training dataset also included 100 synthetic polyp VOIs generated by each method.

After training with data augmentation, the 3D DenseNet was tested with the 12,407 VOIs extracted from the independent test dataset. The likelihoods for the presence of a polyp in a VOI as estimated by the 3D DenseNet were analyzed by use of the pROC package (version 1.16.2) [28] in R (version 3.6.3) [29]. The classification performance was evaluated for the clinically significant polyp size categories of $\geq 6 \mathrm{~mm}$ (all polyps) and 6-9 $\mathrm{mm}$ (small polyps) by use of the AUC as the performance metric. Bootstrapping with 1000 replicates was used for calculation of the $95 \%$ confidence intervals of the AUC values as well as for testing of the difference of the AUC values, where $p<0.01$ indicated a statistically significant difference. 


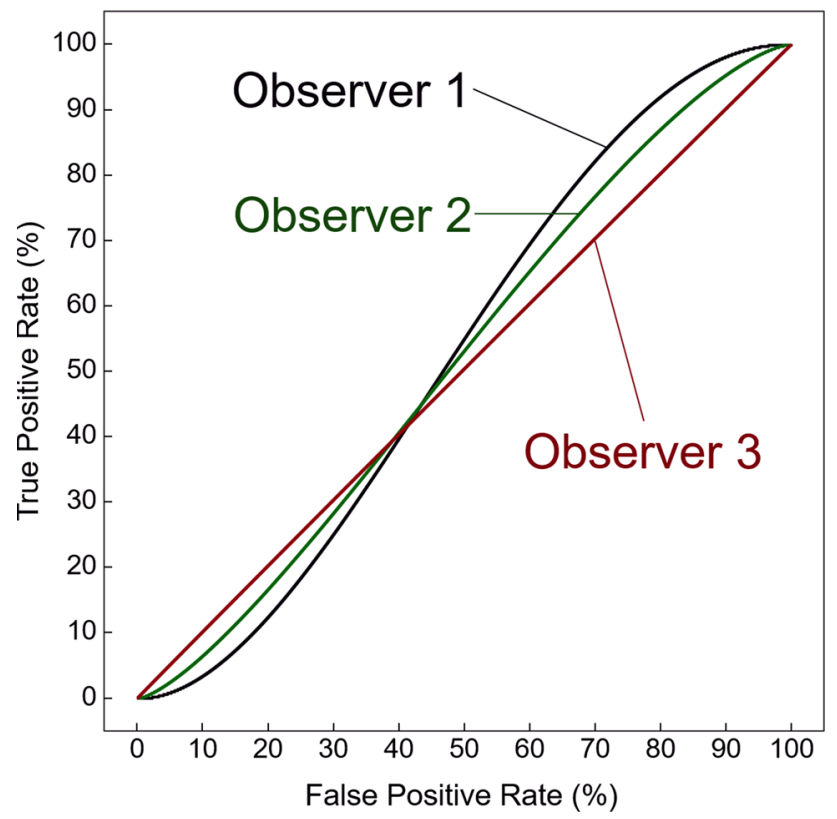

Fig. 6 ROC curves of the three observers for the differentiation between synthetic polyps and real polyps

Table 1 The AUC values, 95\% confidence intervals (CIs) and $p$ values of the three ROC curves in Fig. 6

\begin{tabular}{lll}
\hline & AUC $(\%)(95 \%$ CI $)$ & $p$ value \\
\hline Observer 1 & $52.7[40.7,62.5]$ & 0.65 \\
Observer 2 & $51.9[43.6,60.3]$ & 0.81 \\
Observer 3 & $50.3[42.8,59.1]$ & 0.84 \\
\hline
\end{tabular}

\section{Results}

\section{The observer study}

Figure 6 shows the ROC curves of the performance of the three observers in the differentiation of the 3D Glowgenerated synthetic polyps from real polyps. The ROC curves indicate that the observers were unable to differentiate between the polyps. Table 1 shows an analysis of the AUC values of the ROC curves and their statistical significance with respect to difference from $50 \%$. None of the AUC values of the ROC curves were statistically significantly different from $50 \%(p \geq 0.65)$.

\section{The polyp classification study}

Figure 7 shows the ROC curves representing the classification performance of the 3D DenseNet between polyps and non-polyps based on the training with the different augmentation approaches. The ROC curves on the left show the classification performance for all polyps in our test dataset, whereas the ROC curves on the right show the performance for the clinically significant small polyps $(6-9 \mathrm{~mm}$ in size), the detection of which is generally challenging. In both cases, the use of the 3D Glow-based augmentation approaches, Glow+ (red curves) and Glow (blue curves) yielded a higher performance than did any of the other augmentation approaches, and Glow+ yielded the highest performance.

Table 2 lists the AUC values obtained from the ROC curves in Fig. 7, as well as their pairwise comparison results. The

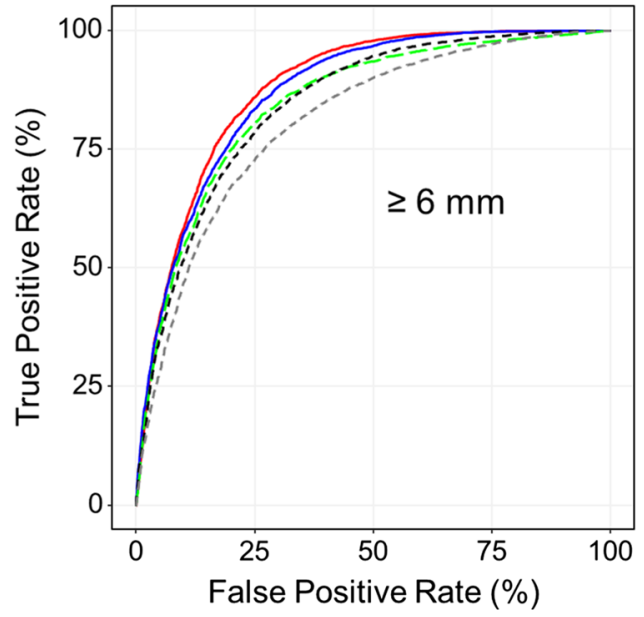

Fig. 7 ROC curves representing the performance of the 3D DenseNet in the classification of polyp candidates with respect to the five different augmentation approaches used for the training step. The left plot shows the ROC curves for all polyps in our test dataset, whereas the right plot shows the ROC curves for clinically significant small polyps. Abbrevia-

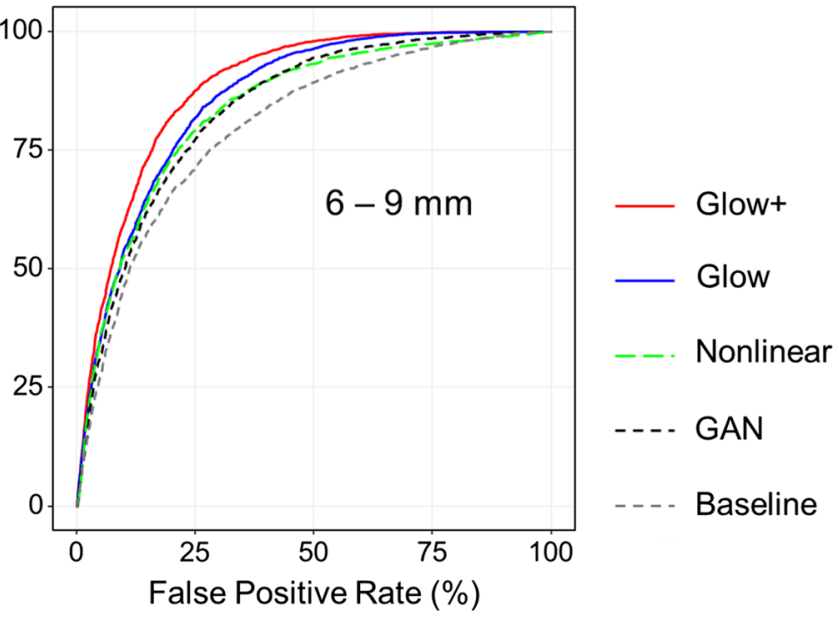

tions Glow $+=3 \mathrm{D}$ Glow with nonlinear augmentation; Glow $=3 \mathrm{D}$ Glow with baseline augmentation; Nonlinear = nonlinear augmentation only; Baseline $=$ baseline augmentation only; GAN $=3 \mathrm{D}$ GAN with baseline augmentation 
Table 2 AUC values obtained from the ROC curves in Fig. 7, and their pairwise comparison results

\begin{tabular}{|c|c|c|c|c|c|}
\hline & Glow+ & Glow & Nonlinear & GAN & Baseline \\
\hline \multicolumn{6}{|l|}{$\geq 6 \mathrm{~mm}$} \\
\hline Glow+ & $88.1[87.5,88.7]$ & 0.00013 & $<0.0001$ & $<0.0001$ & $<0.0001$ \\
\hline Glow & 0.9 & $87.2[86.5,87.8]$ & $<0.0001$ & $<0.0001$ & $<0.0001$ \\
\hline Nonlinear & 3.4 & 2.5 & $84.7[84.1,85.4]$ & 0.49 & $<0.0001$ \\
\hline GAN & 3.5 & 2.6 & 0.1 & $84.6[83.9,85.3]$ & $<0.0001$ \\
\hline Baseline & 7.0 & 6.1 & 3.6 & 3.5 & $81.1[80.3,81.8]$ \\
\hline \multicolumn{6}{|l|}{$6-9 \mathrm{~mm}$} \\
\hline Glow+ & $88.7[88.1,89.2]$ & $<0.0001$ & $<0.0001$ & $<0.0001$ & $<0.0001$ \\
\hline Glow & 2.6 & $86.1[85.6,86.7]$ & $<0.0001$ & $<0.0001$ & $<0.0001$ \\
\hline Nonlinear & 4.6 & 2.0 & $84.1[83.4,84.8]$ & 0.51 & $<0.0001$ \\
\hline GAN & 4.8 & 2.2 & 0.2 & $83.9[83.3,84.6]$ & $<0.0001$ \\
\hline Baseline & 8.3 & 5.7 & 3.7 & 3.5 & $80.4[79.6,81.4]$ \\
\hline
\end{tabular}

The diagonal components show the AUC values in percentage with the 95\% confidence interval in square brackets. The upper triangle shows the $p$ values obtained from a bootstrap test (1000 replicates) for the pairwise comparison of the AUC values. The lower triangle shows the pairwise differences in the AUC values

Glow $+=3 \mathrm{D}$ Glow with nonlinear augmentation; glow $=3 \mathrm{D}$ Glow with baseline augmentation; nonlinear $=$ nonlinear augmentation only; baseline $=$ baseline augmentation only; GAN $=3 \mathrm{D}$ GAN with baseline augmentation

upper table shows the results for all polyps, whereas the lower table shows the results for the clinically significant small polyps (6-9 $\mathrm{mm}$ in size). In both cases, the 3D Glow-based augmentation approaches (Glow+ and Glow) yielded statistically significantly higher AUC values than did the other augmentation approaches, and Glow+ yielded the highest AUC values.

\section{Discussion}

Although deep learning has made it relatively easy to obtain state-of-the-art results of various medical imaging tasks, in CTC, the available training datasets are relatively small, and the numbers of clinically significant polyps are even smaller. In this study, we investigated the feasibility of developing a flow-based generative model for simulating colorectal polyps to improve the training of 3D CNNs in CADe for CTC. The results of our experiments indicate that this is indeed feasible.

In the first experiment, we performed an observer study to compare the visually observed characteristics of 3D Glowgenerated synthetic polyps with those of real polyps. To the best of our knowledge, this is the first study to show that it is possible to generate VOIs of synthetic polyps that are visually equivalent to VOIs of real polyps in CTC.

In the second experiment, we investigated the effect of the training of deep learning (3D DenseNet) with synthetic polyps generated by 3D Glow on the differentiation of polyps from non-polyps in CTC. To the best of our knowledge, this is the first study to show that data augmentation by synthetic polyps can be used to yield a statistically significant performance improvement in deep learning for CTC. We demonstrated that the use of synthetic polyps generated by 3D Glow outperformed the use of traditional nonlinear and GAN-based augmentations, and that the classification performance was highest when 3D Glow was used in combination with a nonlinear augmentation.

The observed improvement in the polyp classification performance by use of the 3D Glow was high for small polyps (6-9 $\mathrm{mm}$ in size). This result is important, because it is the detection of small polyps that remains challenging in CTC, whereas many clinical studies have demonstrated that existing CADe systems can detect large polyps in CTC at a high accuracy $[31,32]$. Therefore, 3D Glow could have a meaningful impact in improving the detection of small polyps.

We performed the polyp detection by use of volumetric analysis of 3D CT colonography data, because this enables deep learning to review the complete region of the colon regardless of obstructions such as collapsed or poorly distended colon segments. Although other methods such as virtual endoscopic views have been used previously for polyp detection by deep learning [6], such approaches are limited to well-distended regions of the colon, whereas obstructed regions that can hide clinically significant polyps or masses would need to be identified and reviewed by other means. Also, the visual appearance of a virtual endoscopic view depends on the rendering algorithm and its visualization parameters, whereas 3D CT colonography data are readily standardized by Hounsfield units. Thus, the volumetric analysis provides the most uniform approach for the application of deep learning to polyp detection in CT colonography. 
This study had several limitations. First, the number of datasets was necessarily relatively small. Second, there are various other data augmentation methods besides the nonlinear image transforms and the GAN-based method that we used as reference augmentation methods in this study. However, a thorough systematic assessment of their comparative performance against 3D Glow will require a separate study. Third, the study could be expanded by consideration of the unique demands of CTC, such as various bowel preparations or polyps that tend to be underrepresented in CTC datasets. Addressing such issues provides topics for future studies.

\section{Conclusion}

We developed a 3D CNN based on a flow-based generative model for generating synthetic colorectal polyps in CTC. Our results indicate that the generated synthetic polyps are visually indistinguishable from real polyps and that data augmentation by such polyps can significantly improve the performance of deep learning in CADe for CTC.

Acknowledgements We thank the Enterprise Research Infrastructure and Services at Mass General Brigham (Boston, MA) for their provision of high-performance computing services.

Funding This study was supported partly by the National Institutes of Health (NIH) under Award Nos. R01CA212382 and R01EB023942. The content is solely the responsibility of the authors and does not necessarily represent the official views of the NIH. J.J.N. and H.Y. are co-inventors of electronic cleansing and computer-aided detection software patents.

\section{Compliance with ethical standards}

Conflict of interest The other authors declare that they have no conflict of interest.

Ethical standards This retrospective single-center study was approved by the Mass General Brigham (MGB) institutional review board (IRB).

Human and animal rights statement All procedures involving human participants were performed in accordance with the ethical standards of the IRB and with the 1964 Declaration of Helsinki and its later amendments.

Informed consent Informed consent was waived for this study by the MGB IRB.

Open Access This article is licensed under a Creative Commons Attribution 4.0 International License, which permits use, sharing, adaptation, distribution and reproduction in any medium or format, as long as you give appropriate credit to the original author(s) and the source, provide a link to the Creative Commons licence, and indicate if changes were made. The images or other third party material in this article are included in the article's Creative Commons licence, unless indicated otherwise in a credit line to the material. If material is not included in the article's Creative Commons licence and your intended use is not permitted by statutory regulation or exceeds the permitted use, you will need to obtain permission directly from the copyright holder. To view a copy of this licence, visit http://creativecomm ons.org/licenses/by/4.0/.

\section{References}

1. Bray F, Ferlay J, Soerjomataram I, Siegel R, Torre L, Jemal A (2018) Global cancer statistics 2018: GLOBOCAN estimates of incidence and mortality worldwide for 36 cancers in 185 countries. CA Cancer J Clin 68:394-424

2. Winawer S, Fletcher R, Miller L, Godlee F, Stolar M, Mulrow C, Woolf S, Glick S, Ganiats T, Bond J, Rosen L, Zapka J, Olsen S, Giardiello F, Sisk J, Van Antwerp R, Brown-Davis C, Marciniak D, Mayer R (1997) Colorectal cancer screening: clinical guidelines and rationale. Gastroenterology 112:594-642

3. U.S. Preventive Services Task Force: Screening for colorectal cancer: U.S. Preventive Services Task Force Recommendation Statement. JAMA 315:2564-2575 (2016)

4. Litjens G, Kooi T, Bejnordi B, Setio A, Ciompi F, Ghafoorian M, van der Laark J, van Ginneken B, Sánchez C (2017) A survey on deep learning in medical image analysis. Med Image Anal 42:60_ 88

5. Shorten C, Khoshgoftaar T (2019) A survey on image data augmentation for deep learning. J Big Data 6:60

6. Näppi J, Hironaka T, Regge D, Yoshida H (2016) Deep transfer learning of virtual endoluminal views for the detection of polyps in CT colonography. In: Proceedings of SPIE medical imaging: computer-aided diagnosis. SPIE Press, vol 97852B, pp V1-8

7. Näppi J, Pickhardt P, Kim D, Hironaka T, Yoshida H (2017) Computer-aided detection of serrated polyps with a deep convolutional neural network in CT colonography. Int J CARS 12S1: 144-146

8. Tachibana R, Näppi J, Ota J, Kohlhase N, Hironaka T, Kim S, Regge D, Yoshida H (2018) Deep learning electronic cleansing for singleand dual-energy CT colonography. Radiographics 38:2034-2050

9. Chen Y, Ren Y, Fu L, Xiong J, Larsson R, Xu X, Sun J, Zhao J (2018) A 3D convolutional neural network framework for polyp candidates detection on the limited dataset of CT colonography. In: Conf Proc IEEE Eng Med Biol Soc. Honolulu, HI, USA, pp 678-681

10. Näppi J, Hironaka T, Yoshida H (2017) Detection of colorectal masses in CT colonography: application of deep residual networks for differentiating masses from normal colon anatomy. In: SPIE medical imaging: computer-aided diagnosis. Houston, Texas, United States, vol 10575, p 1057518

11. Roth H, Lu L, Liu J, Yao J, Seff A, Cherry K, Kim L, Summers $\mathrm{R}$ (2016) Improving computer-aided detection using convolutional neural networks and random view aggregation. IEEE Trans Med Imaging 35:1170-1181

12. Uemura T, Lu H, Kim H, Tachibana R, Hironaka T, Näppi J, Yoshida H (2018) 3D deep residual convolutional networks for computer-aided detection of polyps in CT colonography. Int $\mathrm{J}$ CARS 13S1:94-95

13. Yi X, Walia E, Babyn P (2019) Generative adversarial network in medical imaging: a review. Med Image Anal 58:101552

14. Shin HC, Tenenholtz NA, Rogers JK, Schwarz CG, Senjem ML, Gunter JL, Andriole KP, Michalski M (2018) Medical image synthesis for data augmentation and anonymization using generative adversarial networks. In: Gooya A, Goksel O, Oguz I, Burgos $\mathrm{N}$ (eds) Simulation and synthesis in medical imaging. Springer, Cham, pp 1-11

15. Uemura T, Näppi J, Lu H, Tachibana R, Hironaka T, Yoshida H (2019) Ensemble 3D residual network (E3D-ResNet) for reduction 
of false-positive polyp detections in CT colonography. In: Proc SPIE medical imaging 2019: computer-aided diagnosis, p 1095038

16. Kingma D, Prafulla D (2018) Glow: generative flow with invertible $1 \times 1$ convolutions. In: Bengio S, Wallach $\mathrm{H}$, Larochelle $\mathrm{H}$, Grauman K, Cesa-Blanchl N, Garnett R (eds) 32nd conference on neural information processing systems (NIPS), Montreal, Canada, pp 10235-10244

17. Dinh L, Sohl-Dickstein J, Bengio Y (2017) Density estimation using real NVP. In: 5th International conference on learning representations (ICLR)

18. Dinh L, Krueger D, Bengio Y (2014) NICE: Non-linear independent components estimation. Technical report

19. Pickhardt P, Choi J, Hwang I, Butler J, Puckett M, Hildebrandt H, Wong R, Nugent P, Mysliwiec P, Schindler W (2003) Computed tomographic virtual colonoscopy to screen for colorectal neoplasia in asymptomatic adults. N Engl J Med 349:2191-2200

20. Rockey DC, Paulson E, Niedzwiecki D, Davis W, Bosworth HB, Sanders L, Yee J, Henderson J, Hatten P, Burdick S, Sanyal A, Rubin DT, Sterling M, Akerkar G, Bhutani MS, Binmoeller K, Garvie J, Bini EJ, McQuaid K, Foster WL, Thompson WM, Dachman A, Halvorsen R (2005) Analysis of air contrast barium enema, computed tomographic colonography, and colonoscopy: prospective comparison. Lancet 365(9456):305-311

21. Johnson CD, Chen MH, Toledano AY, Heiken JP, Dachman A, Kuo MD, Menias CO, Siewert B, Cheema JI, Obregon RG, Fidler JL, Zimmerman P, Horton KM, Coakley K, Iyer RB, Hara AK, Halvorsen RA, Casola G, Yee J, Herman BA, Burgart LJ, Limburg PJ (2008) Accuracy of CT colonography for detection of large adenomas and cancers. N Engl J Med 359(12):1207-1217

22. Regge D, Laudi C, Galatola G, Della Monica P, Bonelli L, Angelelli G, Asnaghi R, Barbaro B, Bartolozzi C, Bielen D, Boni L, Borghi C, Bruzzi P, Cassinis MC, Galia M, Gallo TM, Grasso A, Hassan C, Laghi A, Martina MC, Neri E, Senore C, Simonetti G, Venturini S, Gandini G (2009) Diagnostic accuracy of computed tomographic colonography for the detection of advanced neoplasia in individuals at increased risk of colorectal cancer. JAMA 301(23):2453-2461

23. Nagata K, Okawa T, Honma A, Endo S, Kudo SE, Yoshida H (2009) Full-laxative versus minimum-laxative fecal-tagging CT colonography using 64-detector row CT: prospective blinded comparison of diagnostic performance, tagging quality, and patient acceptance. Acad Radiol 16(7):780-789
24. Näppi J, Yoshida H (2007) Fully automated three-dimensional detection of polyps in fecal-tagging CT colonography. Acad Radiol 25:287-300

25. Yoshida H, Näppi J (2001) Three-dimensional computer-aided diagnosis scheme for detection of colonic polyps. IEEE Trans Med Imaging 20:1261-1274

26. Näppi J, Yoshida H (2008) Adaptive correction of the pseudoenhancement of CT attenuation for fecal-tagging CT colonography. Med Image Anal 12:413-426

27. Näppi J, Yoshida H (2002) Automated detection of polyps in CT colonography: evaluation of volumetric features for reduction of false positives. Acad Radiol 9:386-397

28. Robin X, Turck N, Hainard A, Tiberti N, Lisacek F, Sanchez JC, Mller M (2011) pROC: an open-source package for R and S+ to analyze and compare ROC curves. BMC Bioinform 12:77

29. R Core Team: R: a language and environment for statistical computing. R Foundation for Statistical Computing, Vienna, Austria (2020) https://www.R-project.org/

30. Uemura T, Näppi J, Hironaka T, Hyougseop K, Yoshida $H$ (2020) Comparative performance of 3D-DenseNet, 3D-ResNet, and 3D-VGG models in polyp detection for CT colonography. In: Proceedings of SPIE medical imaging 2020: computer-aided diagnosis, p 1131435. https://doi.org/10.1117/12.2549103

31. Regge D, Halligan S (2013) CAD: How it works, how to use it, performance. Eur J Radiol 82:1171-1176

32. Näppi J, Uemura T, Kim S, Kim H, Yoshida H (2020) Comparative performance of 3D machine-learning and deep-learning models in the detection of small polyps in dual-energy CT colonography. In: Proceedings of SPIE medical imaging 2020: computer-aided diagnosis, p 113143C. https://doi.org/10.1117/12.2549793

Publisher's Note Springer Nature remains neutral with regard to jurisdictional claims in published maps and institutional affiliations. 